\title{
MECHANISM OF CARRIER HEATING IN HIGH ELECTRIC FIELD ELECTROLUMINESCENCE DEVICES*
}

\author{
S. Dedulewicz ${ }^{\dagger}$ and M. Godlewski \\ Institute of Physics, Polish Academy of Sciences \\ Al. Lotników 32/46, 02-668 Warszawa, Poland
}

\begin{abstract}
The mechanism of carrier heating in $\mathrm{ZnS}$-based electroluminescence devices is discussed. We show that carrier acceleration by an applied electric field is at $300 \mathrm{~K}$ not a loss-free (ballistic carriers) process. Even for large carrier energies (about $2.5 \mathrm{eV}$ ) inelastic scattering on polar optical phonons dominates over elastic scattering on acoustic phonons and ionized impurities.
\end{abstract}

PACS numbers: 72.10.-d, 78.60.Fi

\section{Introduction}

Seemingly the most important questions on the mechanisms of rare earth or transition metal ions excitation and on free carriers acceleration in high electric field electroluminescence (EL) devices remain still partly unanswered. It is still disputed if carrier acceleration in ZnS EL is loss-free (ballistic carriers) or, in contrary, carriers are initially "cold" but can gain sufficient energy for impact processes from the applied electric field. It was claimed that the relatively high efficiency of $\mathrm{ZnS}$ and alkaline earth sulfides based EL devices is due to their unique properties of a loss-free acceleration. EL is induced by generation of electrons tunnel-emitted from interface states by an electric field higher than the one needed for ballistic acceleration [1, 2].

In this paper we present the theoretical evaluation of the carrier heating process by an applied electric field. We show that, depending on the dominant carrier scattering mechanism, the initially "cold" free electrons can reach the threshold energy for impact processes. The efficiency of such a carrier heating critically depends on the material parameters, magnitude of the applied electric field, doping level (via scattering on ionized impurities) and on lattice temperature.

\footnotetext{
*This work was partly supported by the grant No. 204769101 of the Committee for Scientific Research.

tOn leave from Semiconductor Physics Institute, A. Goštauto 11, Vilnius, Lithuania.
} 


\section{Results and discussion}

Theoretical calculations of the mean electron energy at low and high electric fields in the electroluminescence of $\mathrm{ZnS}$ were performed by the Monte Carlo method taking into account the following energy and momentum dissipation mechanisms:

- inelastic (at low temperature) and elastic (at high temperature) scattering on acoustic phonons (as described in Ref. [3]),

- piezoelectric scattering (as described in Ref. [4]),

- polar optical phonon scattering (as described in Ref. [5]),

- scattering on ionized impurities (as described in Ref. [3]).

In the case of phonon related processes the scattering rates depend on the mean electron energy [3,5] (Fig. 1). It was also taken into account that the Debye screening length depends on temperature. This length was arbitrarily assumed to be equal at low temperature half of the mean distance between impurities (similar approximation was used in Ref. [1]). Concentration of shallow ionized impurities was taken as $10^{16} \mathrm{~cm}^{-3}$, which is a typical contamination level in the case of the $\mathrm{ZnS}$ material.

Figure 1 presents the dependence of scattering probability for the different mechanisms listed above on the mean electron energy. The results shown in Fig. 1 were calculated for $300 \mathrm{~K}$ and were obtained for material parameters (acoustic deformation potential, static and dynamic dielectric constant, Debye temperature, effective mass of electron, sound velocity and material density) of the cubic of $\mathrm{ZnS}$ crystals. At $300 \mathrm{~K}$ and the electron energy about $2.5 \mathrm{eV}$ (required for impact excitation of typical electroluminescence activators) the scattering is still dominated by that on optical phonons, which is two times more probable than elastic scattering on acoustic phonons. This is the important result which may solve the long dispute on the carrier loss mechanism in the $\mathrm{EL}$ of $\mathrm{ZnS}$ [4]. It was claimed, as mentioned in the Introduction, that electron acceleration in $\mathrm{ZnS}$ has a loss-free character and that this fact accounts for the high efficiency of ZnS:Mn EL [4]. In accordance with the results shown in Fig. 1, a loss-free acceleration of electrons is not expected.

In Figure 2 we show the time dependence of the mean electron energy for different electric fields applied. We show here that even for relatively low electric fields $10^{5} \mathrm{~V} \mathrm{~cm}^{-1}$ fields are used to obtain a high quantum efficiency of EL [5]) some of the electrons can gain an energy sufficient for impact excitation of EL activators in the ps time range. This is not the case for carrier heating at low temperature, due to a very rapid increase of the probability of acoustic phonon emission with a rise in the mean electron energy. For low electric fields, in the range of a few $\mathrm{kV} \mathrm{cm}^{-1}$, the mean electron energy saturates very fast at meV values, i.e. far from energies sufficient for impact excitation. Here, much larger electric fields are required to reach the threshold for EL excitation.

We have also performed Monte Carlo simulations of electron heating dynamics to determine the dependence of the electron mean energy on heating time for a given electric field. Calculations of electron energy distribution for different electric fields applied are presently on the way. The first results obtained indicate that 


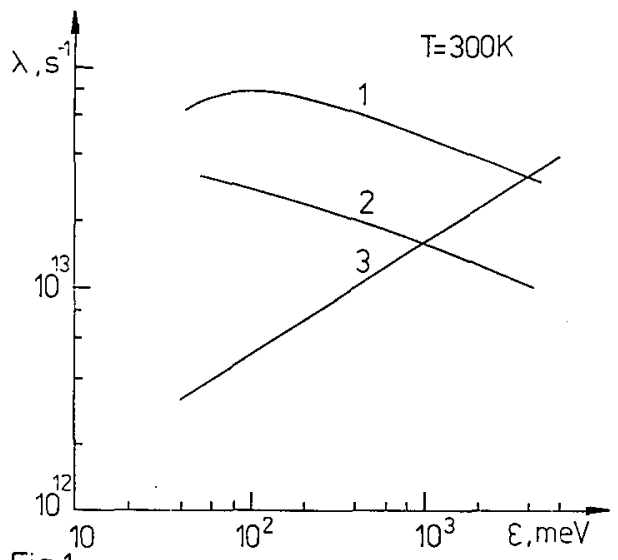

Fig.1

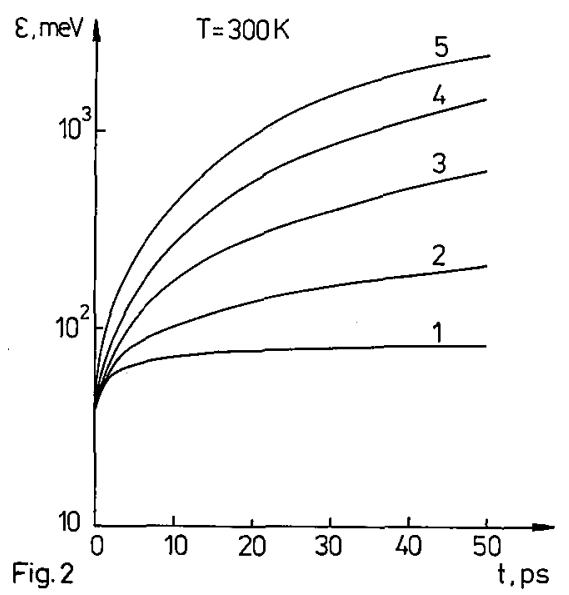

Fig. 1. Energy dependence of scattering rates $(\lambda)$ for optical phonon emission (1), optical phonon absorption (2) and elastic acoustic phonons (3) for $300 \mathrm{~K}$ and material parameters of $\mathrm{ZnS}$. The nonelastic scattering processes are still dominant for mean electron energy smaller than about $4 \mathrm{eV}$.

Fig. 2. The time dependence of the mean electron energy for five different electric fields applied: 1 (1), $1.5(2), 2(3), 2.5(4)$ and $3(5) \mathrm{kV} \mathrm{cm}^{-1}$ and $300 \mathrm{~K}$ lattice temperature.

the electron energy distribution is for low electric fields a relatively narrow function which broadens with the increase in electric field magnitude. The main aim of these calculations is to explain relatively sharp thresholds for center excitation (ionization) observed in experiments.

\section{References}

[1] R. Mach, G.O. Müller, G. Schulz, J. von Kalben, W. Gericke, Phys. Status Solidi A 81, 733 (1984).

[2] G.O. Müller, Acta Polytech. Scand. Appl. Phys. Ser. 170, 13 (1990).

[3] C. Canali, C. Jacobani, F. Nava, G. Otta viani, A. Alberigi-Qvaranta, Phys. Rev. B 12, 2265 (1975).

[4] D.L. Rode, Phys. Rev. B 2, 1012 (1970).

[5] W. Fawcett, A.D. Boardman, S. Swain, J. Phys. Chem. Solids 31, 1963 (1970).

[6] G.O. Müller, R. Mach, Phys. Status Solidi A 77, K179 (1983).

[7] J.W. Allen, J. Lumin. 31/32, 665 (1984). 